This item was submitted to Loughborough's Research Repository by the author.

Items in Figshare are protected by copyright, with all rights reserved, unless otherwise indicated.

\title{
Evaluation of a subject-specific, torque-driven computer simulation model of one-handed tennis backhand ground strokes
}

\section{PLEASE CITE THE PUBLISHED VERSION}

http://journals. humankinetics.com/jab-current-issue/jab-volume-27-issue-4-november/evaluation-of-a-subjectspecific-torque-driven-computer-simulation-model-of-one-handed-tennis-backhand-ground-strokes

\section{PUBLISHER}

(C) Human Kinetics

\section{VERSION}

VoR (Version of Record)

\section{LICENCE}

CC BY-NC-ND 4.0

\section{REPOSITORY RECORD}

Kentel, Behzat B., Mark A. King, and Sean R. Mitchell. 2019. "Evaluation of a Subject-specific, Torque-driven Computer Simulation Model of One-handed Tennis Backhand Ground Strokes”. figshare.

https://hdl.handle.net/2134/9379. 
This item was submitted to Loughborough's Institutional Repository (https://dspace.lboro.ac.uk/) by the author and is made available under the following Creative Commons Licence conditions.

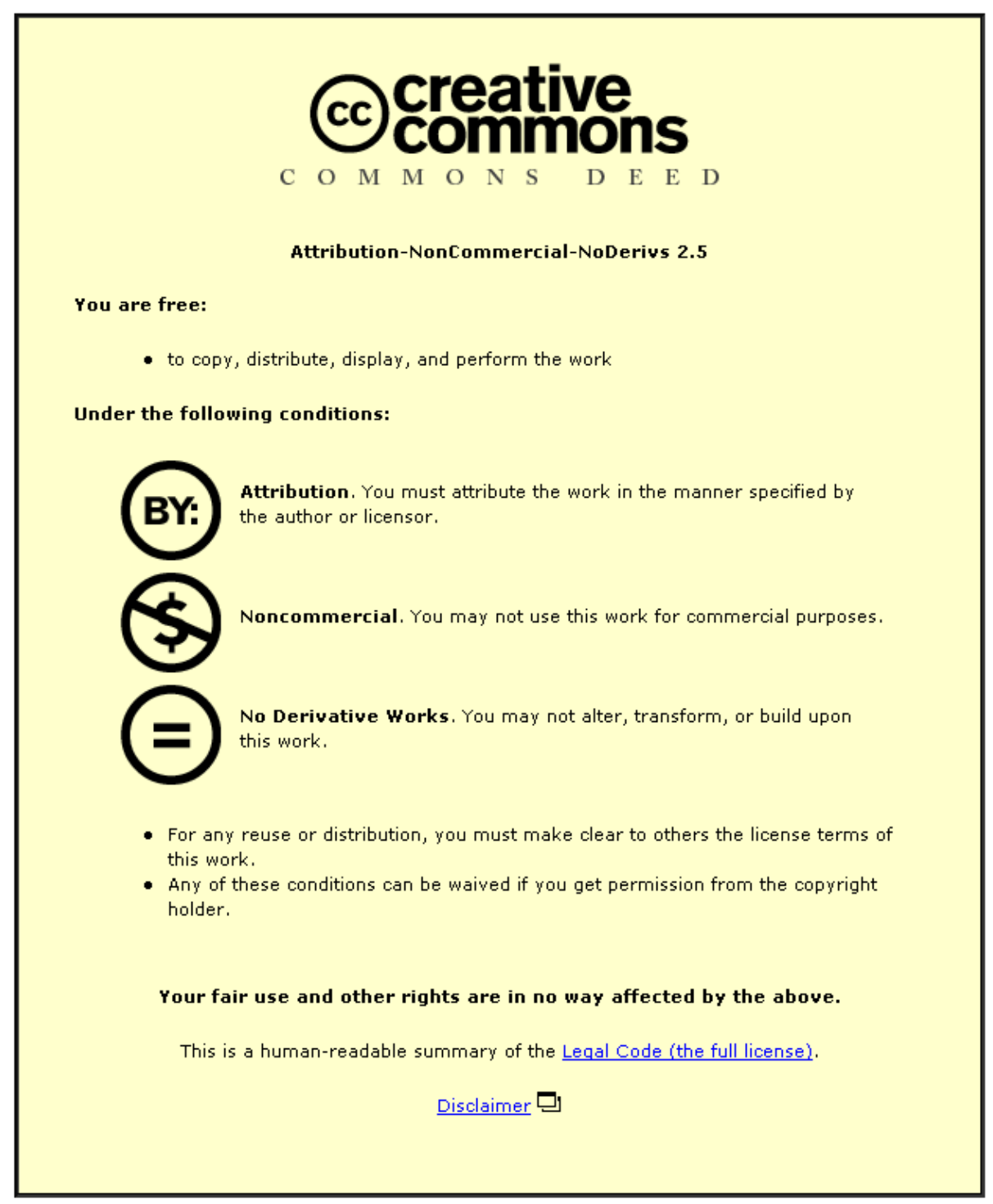

For the full text of this licence, please go to: http://creativecommons.org/licenses/by-nc-nd/2.5/ 


\title{
Evaluation of a Subject-Specific, Torque-Driven Computer Simulation Model of One-Handed Tennis Backhand Ground Strokes
}

\author{
Behzat B. Kentel, Mark A. King, and Sean R. Mitchell \\ Loughborough University
}

\begin{abstract}
A torque-driven, subject-specific 3-D computer simulation model of the impact phase of one-handed tennis backhand strokes was evaluated by comparing performance and simulation results. Backhand strokes of an elite subject were recorded on an artificial tennis court. Over the 50-ms period after impact, good agreement was found with an overall RMS difference of $3.3^{\circ}$ between matching simulation and performance in terms of joint and racket angles. Consistent with previous experimental research, the evaluation process showed that grip tightness and ball impact location are important factors that affect postimpact racket and arm kinematics. Associated with these factors, the model can be used for a better understanding of the eccentric contraction of the wrist extensors during one-handed backhand ground strokes, a hypothesized mechanism of tennis elbow.
\end{abstract}

Keywords: off-center impacts, racket and arm kinematics, arm-racket system

Computer simulation models, representing the whole or a part of the body in specific movements in sports, can be used to help understand the causes of injuries and to search for optimum technique or optimum equipment parameters to enhance performance (Yeadon et al., 1990; King et al., 2006; Neptune et al., 2009). In tennis, computer simulation has been used to investigate the effects of racket inertia on elbow loading and racket behavior for center and off-center ball impacts during a forehand stroke (Nesbit et al., 2006). In addition, a one-handed backhand computer simulation model has been used to compare the effects of impact location, stringbed tension, racket frame inertia, and stiffness on elbow loading (Glynn et al., 2007).

One particular issue affecting nearly half of all tennis players is elbow pain (Carroll, 1981), with the most common injury being lateral epicondylitis, or tennis elbow (Giangarra et al., 1993; Kelley et al., 1994), which occurs in the wrist extensor muscle tendons where they attach to the lateral epicondyle of the humerus. It is likely that eccentric contraction of the wrist extensor muscles during the one-handed backhand ground stroke is a key

Behzat B. Kentel is with the Wolfson School of Mechanical and Manufacturing Engineering, and with the School of Sport, Exercise and Health Sciences, Loughborough University, Leicestershire, U.K. Mark A. King (Corresponding Author) is with the School of Sport, Exercise and Health Sciences, Loughborough University, Leicestershire, U.K. Sean R. Mitchell is with the Wolfson School of Mechanical and Manufacturing Engineering, Loughborough University, Leicestershire, U.K. injury mechanism for tennis elbow (Blackwell \& Cole, 1994; Knudson, 2004) with increased extensor activity found during ball-racket impact for players with tennis elbow (Kelley et al., 1994; Bauer \& Murray, 1999). Furthermore, novice tennis players incur a greater incidence of tennis elbow and generally tend to execute the onehanded backhand ground stroke with a flexed wrist and a wrist flexion angular velocity at the instant of ball-racket impact (Blackwell \& Cole, 1994; Riek et al., 1999).

Ball impact location and grip tightness also influence the amount of eccentric contraction of the wrist extensors and by association tennis elbow, with the hand tending to rotate with the racket around the wrist for off-center impacts (Roetert et al., 1995; Knudson \& Blackwell, 1997). For off-center impacts, higher levels of elbow loading for backhand strokes have been found when compared with center impacts (Hennig et al., 1992). In addition, Knudson (1991) showed that the postimpact loading on the hand for forehand strokes was highly influenced by the ball impact location. For grip tightness, (Hatze, 1976) reported that a reduction of 10-15\% in the magnitude of the vibrations measured on the racket frame with a loose grip compared with a tight grip. Furthermore, it has been recognized that a rigid connection between the hand and racket is not consistent with experimental findings (Brody, 1989; Hatze, 1992; Kawazoe, 1997; Knudson, 1997; Hennig, 2007).

There are clearly a number of factors that influence the amount of eccentric contraction of the wrist extensors, but experimentally it is difficult to control each factor and measure directly the level of eccentric contractions during tennis strokes. Computer simulation offers 
an alternative approach, giving complete control and allowing individual factors to be investigated, although for a computer simulation to be useful it is vital that the model is evaluated by comparing simulations with performances; otherwise, the results obtained might be misleading (Yeadon \& Challis, 1994). For a simulation model of one-handed tennis backhand strokes, both center/off-center ball impacts and gripping the racket should be considered within the evaluation process, as these are key factors in determining the racket and arm motion during and after ball impact.

The purpose of this study was to develop and evaluate a subject-specific, torque-driven 3-D computer simulation model capable of simulating ball impacts at a range of locations on the stringbed with a view to investigating under what conditions there are higher levels of eccentric contraction of the wrist extensors during one-handed tennis backhand ground strokes and potentially tennis elbow.

\section{Methods}

A nine-segment simulation model was built using MSC. ADAMS (MSC.Software Corp., California, USA) and consisted of a torso, humerus, ulna, radius, hand, racket handle, and racket head, along with two additional elements representing upper-arm and forearm wobbling masses (Figure 1). The upper-arm and forearm wobbling masses were connected to the proximal and distal ends of the bone segments with nonlinear spring-damper systems,

$$
F=-k x^{3}-c \dot{x}
$$

where $k$ and $c$ are stiffness and damping coefficient, respectively, and $x$ equals the distance between wobbling mass and bone (Pain \& Challis, 2001). Nine point masses were used to represent the stringbed and the ball was modeled as a rigid sphere that could contact the racket at any of the nine point mass locations (Figure 1). The impact of ball and racket was modeled as a normal impact force and a frictional force perpendicular and tangential to the racket head plane, respectively (Glynn et al., 2007).

The kinematic chain from torso to racket head had 12 rotational degrees of freedom: three at the shoulder, two at the elbow (flexion / extension and pronation / supination), two at the wrist (flexion / extension and radial / ulnar deviation), three at the grip, and two between the racket handle and racket head. In addition, the translation and orientation of the torso were input to the model from performance data collected (see below). The joints in the arm were formed by coincident sequential revolute joints allowing rotations in the sagittal, frontal, and transverse planes, respectively. To achieve realistic pronation/supination movement of the forearm, the rotation axis normal to the transverse plane at the elbow joint was tilted to pass through the center of the radial head and the center of the distal end of the ulna (Morrey \& Chao, 1976). A ball and socket joint was used to connect the hand and the racket. Seven pairs of torque generators (flexor and extensor) were used to control the joint angle changes

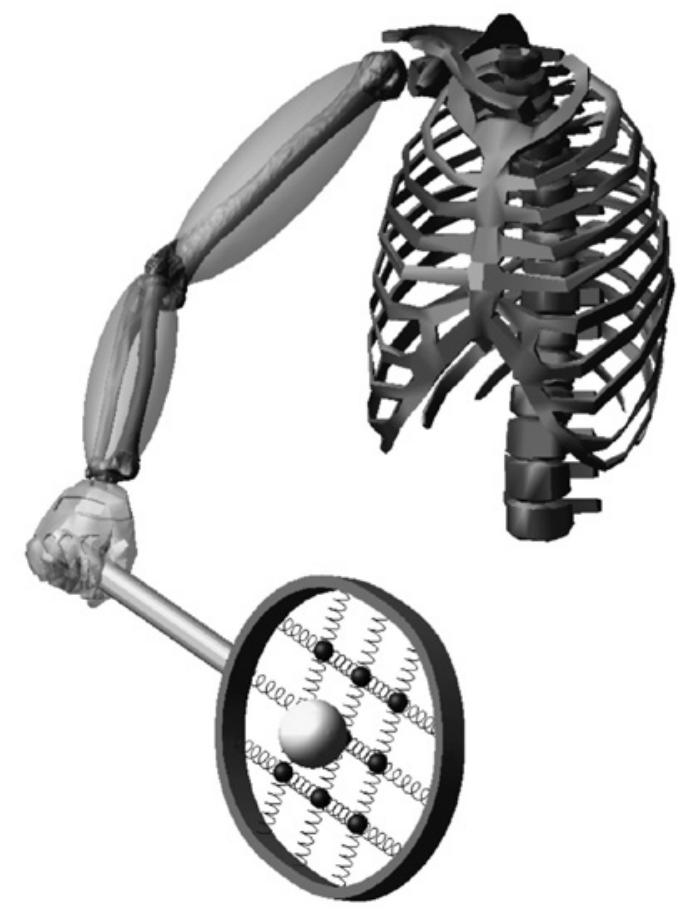

Figure 1 - Computer simulation model of one-handed tennis backhand strokes including the tennis ball.

in the model: three pairs at the shoulder, two pairs at the elbow, and two pairs at the wrist. In addition, three pairs of equal and opposite torques located between the hand and the racket were used to represent the variation in gripping torque throughout the stroke around the principal axes of the tennis racket. The gripping torque before impact was represented as a function of time. During and after the impact, three torsional springs around the three principal axes of the racket were used to represent the resistance of the racket motion within the hand. Simulations started at the beginning of the racket's forward motion at the start of tennis stroke and ended $50 \mathrm{~ms}$ after ball-racket impact when the effects of the impact on the wrist and elbow kinematics have returned to their preimpact magnitudes (Glynn et al., 2007).

The simulation model was customized to simulate one-handed tennis backhand strokes performed by a 22-year-old male elite tennis player (ranked in U.K. top 20), who gave informed consent for the procedures during data collection sessions as approved by the Loughborough University Ethical Advisory Committee. New Pro Penn Titanium tennis balls were fired from a Bola ball cannon at $30 \pm 1 \mathrm{~m} / \mathrm{s}$ and after a warm-up period, the subject was asked to perform one-handed topspin backhand strokes. The performances were recorded by a Vicon 624 System (OMG plc, Oxford, U,K.) with 12 M2 strobe cameras operating at $250 \mathrm{~Hz}$. Thirty-three $25-\mathrm{mm}$ retro-reflective markers were attached to the subject's body (standard marker set) and six markers were attached to the tennis racket (Figure 2). Two genlocked Phantom 


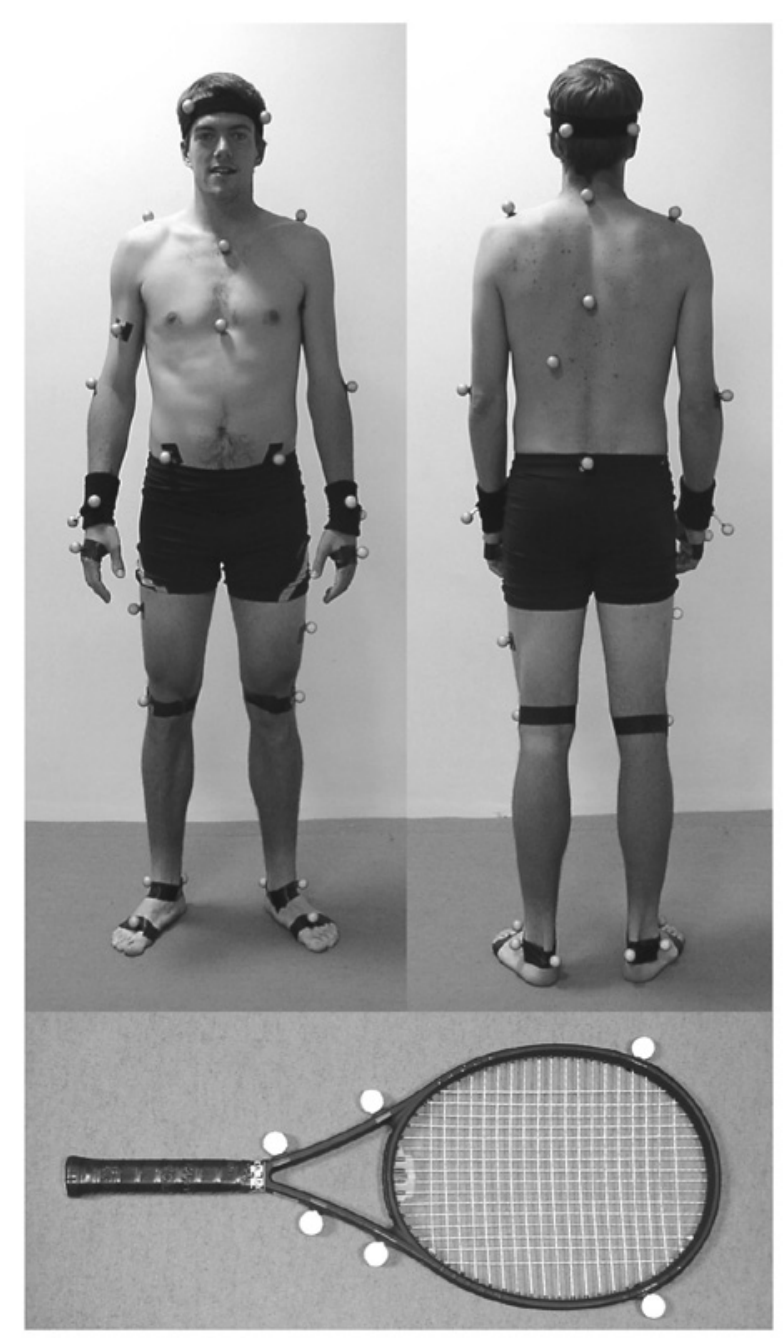

Figure 2 - Elite subject and the tennis racket with retroreflective markers shown.

V4.1 high-speed digital cameras operating at $2500 \mathrm{~Hz}$ and located behind and to the side of the subject were synchronized with the Vicon system and were used to measure the ball velocity at impact, impact location, impact time, and ball contact period.

Two typical backhand strokes, one with the tennis ball contacting the center of the stringbed and the other with the ball contacting off-center (below the longitudinal axis of the racket near the tip of the racket) were selected. Since the center impact and off-center impact strokes were collected in the same data collection session with the subject trying to perform the same stroke, the expectation was that the changes in the motion of the racket and arm during the 50-ms period impact onward would be primarily due to the difference in ball impact location rather than differences in technique, as there is insufficient time to react to an off-center ball impact location (Lewis \& Brown, 1994; de Rugy \& Sternad, 2003). The segmental motions of the arm and racket were calculated using Euler angles between the proximal and distal segment reference frames with a sagittal, frontal, and the transverse plane sequence of rotations. The neutral position of each segment was chosen to be the anatomical position of the thorax and arm. Raw angle data were smoothed by fitting quintic splines (Wood \& Jennings, 1979). To avoid oversmoothing the impact, the raw racket and wrist angle data sets were divided into two subsets at the time of impact (with separate splines fitted to each subset) —one containing data before initial contact and the other data after initial contact, with each data set sharing a common point at initial ball racket contact.

A Cybex Norm isovelocity dynamometer was used to measure maximum voluntary joint torques around the shoulder, elbow, and wrist joints of the subject. Maximum voluntary isometric torques were measured for eight joint angles spanning the range of motion for each joint movement. Isovelocity trials were collected at seven different angular velocities of the crank $(50,100,150,200,250$, 300 , and $350 \%$ s) using a two-repetition concentric-eccentric exercise protocol (King \& Yeadon, 2002). Ninetyfive anthropometric measurements for calculating the segment inertia parameters were taken from the subject (Yeadon, 1990). In addition, 3-D surface bone meshes of the subject's right arm (i.e., humerus, radius, and ulna) were generated from MRI using the Mimics software (Materialise UK, Sheffield, U.K.). The program MSC. ADAMS was then used to calculate inertia parameters of the humerus, ulna, and radius using the geometry of the bones from MRI and bone densities based upon the literature (Clarys \& Marfell-Jones, 1986; Pluim et al., 2007). The inertia parameters of the wobbling masses were determined so that the combined system of bone and wobbling mass was inertially equivalent to the whole segment. The nonlinear stiffness coefficients for the wobbling masses were taken from a previous study (Glynn et al., 2007), and near-critical damping coefficients (Pain $\&$ Challis, 2001) were determined by simulating the movement of the wobbling masses when the arm was fixed in the anatomical position. The inertia and elastic parameters of the racket frame, viscoelastic parameters of the stringbed, and viscoelastic parameters of the tennis ball for normal and oblique impacts were taken from a previous study in which the same racket/ball was used (Glynn et al., 2007).

The torque generators at the joints consisted of a rotational contractile component and an elastic component in series (King et al., 2006). The series elastic component torque was calculated as the product of an elastic component angle and a stiffness parameter determined assuming $5 \%$ stretch for a maximal isometric contraction (de Zee \& Voigt, 2001). The maximum voluntary contractile component torque (function of contractile angle and angular velocity) was obtained from a nine-parameter function. Seven of the nine parameters defined the maximum voluntary torque as a function of contractile component angular velocity (Yeadon et al., 2006) and the remaining two parameters were used to include the contractile angle dependence as a quadratic function of angle (King et al., 
2006). The torque parameters were determined by minimizing the RMS difference between the nine-parameter function and measured torque values using the simulated annealing algorithm (Corana et al., 1987).

The torque exerted by each torque generator during a simulation was equal to the maximum voluntary torque multiplied by a torque activation level for that torque generator. The activation level was specified as a function of time and defined what proportion of maximum was exerted at a joint at a particular time, where 0 corresponds to no activation and 1 corresponds to full activation. Two profiles representing the activation of the flexor and extensor torque generators were defined using six activation parameters for each of them (Figure 3). The general extensor profile started with an initial preactivation level to represent the activation before the start of the simulation. During the swing, the activation level ramped up and reached a peak activation level before impact using a quintic function (Yeadon \& Hiley, 2000). After impact, the activation level ramped down to zero. The general flexor profile started from an

a

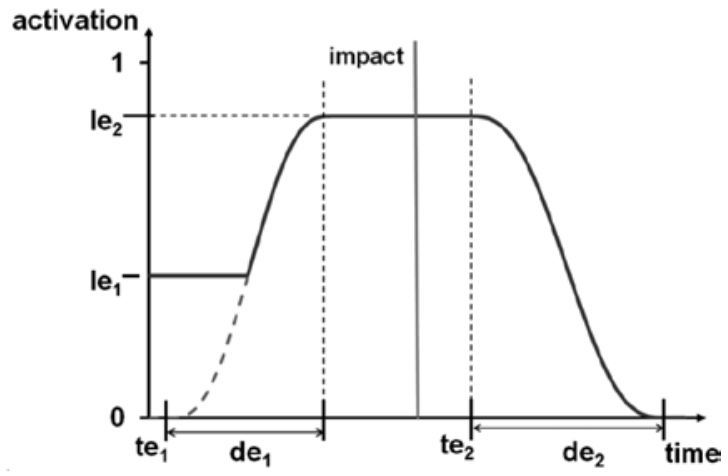

b

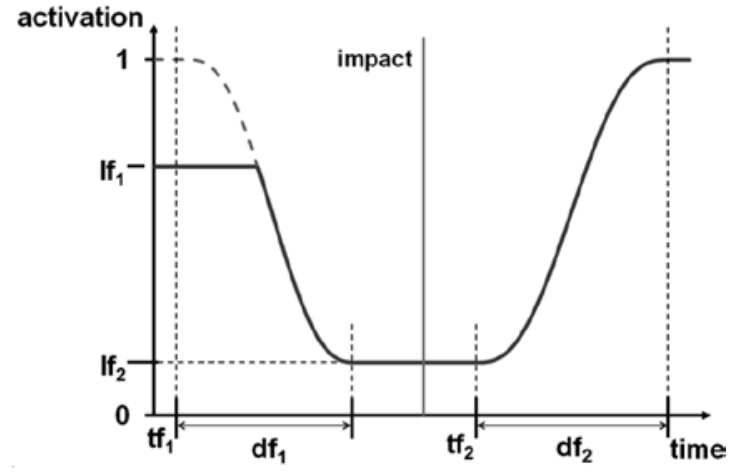

Figure 3 - (a) Torque activation profiles for extensors: $\mathrm{le}_{1}$ is the initial activation level, $\mathrm{l}_{2}$ is the maximum activation level, $t_{1}$ is the start time of the ramping up from zero activation, de $e_{1}$ is the duration of the ramping up, te $e_{2}$ is the start time of the ramping down to zero activation, and $\mathrm{de}_{2}$ is the duration of the ramping down. (b) Torque activation profiles for flexors: $\mathrm{lf}_{1}$ is the initial activation level, $\mathrm{lf}_{2}$ is the minimum activation level, $\mathrm{tf}_{1}$ is the start time of the ramping down from full activation, $\mathrm{df}_{1}$ is the duration of the ramping down, $\mathrm{tf}_{2}$ is the start time of the ramping up to full activation, and $\mathrm{df}_{2}$ is the duration of the ramping up. initial preactivation level and ramped down to a minimum level before impact using a quintic function. After impact, the activation level increased to 1 (full activation) during the backhand stroke. Of the 14 torque generators (seven pairs), shoulder adductor, shoulder internal rotator, elbow flexor, and pronator used the flexor profile, whereas extensor profiles were used for the others. The activation profile type for each torque generator was selected by considering the joint motion as well as observed muscle activation in the literature (Chow et al., 1999). For the profiles of wrist extensors and flexors maximum activations were permitted before impact, as initial simulations showed that this was needed and it agreed with data in the literature.

The time histories of the gripping torque components before impact were estimated using quintic functions similar to the torque activation profiles described earlier. Each component of the gripping torque started with an initial torque level and either ramped up or down during the swing before impact. The upper bound for the gripping torques were estimated from previous studies (McLaughlin \& Miller, 1980; Glynn et al., 2007) and the lower bounds were set to zero. During and after ball impact, the resistance of the racket motion within the hand was represented by three torsional spring dampers around the axes parallel to the principal axes of the racket at the grip (neutral position at ball impact).

The simulation model was evaluated by matching the center impact trial. To closely match the conditions at the time of ball impact, the complete backhand swing was divided into two phases: preimpact and impact onward. The preimpact phase was matched to obtain the values of the kinematic variables and the activation levels at the instant of ball-racket impact. The impact-onward simulations started at impact using the conditions at impact obtained from the optimized preimpact simulation. A total of 74 parameters (62 torque activation and 12 grip activation parameters) were determined during the preimpact phase matching. Initially, the RMS difference between performance and simulation in terms of joint angles and racket angles was calculated for the whole preimpact period. Once a satisfactory match was obtained, the solution was refined by reoptimizing using the RMS difference over the last $50 \mathrm{~ms}$ of the preimpact phase to give a close match at the critical instant of ball impact. The $50 \mathrm{~ms}$ impact-onward phase was then matched by varying the 40 parameters ( 28 torque activation and 12 grip parameters) to minimize the RMS difference between simulation and performance. The ball impact location in the initial matching simulation was then perturbed to be the same as that in the off-center trial and a single simulation (using the center trial matching parameters) was compared with the recorded off-center trial in terms of range of motion and movement characteristics for wrist flexion and racket rotation about its longitudinal axis. Considering the off-center impact simulation, upper bounds for the grip parameters were adjusted (reduced) to allow more racket rotation relative to the hand since the racket rotations in the simulation were found to be much lower than actual rotations. The impact-onward 
matching process was then repeated to give a solution that matched the center impact trial while also giving a realistic simulation when the ball impact location was perturbed to be off center.

\section{Results}

Measurements on the elite tennis player allowed subjectspecific inertia parameters (Table 1) and subject-specific torque parameters (Table 2, Figure 4) to be determined. The nonlinear stiffness coefficients for the springs attaching the upper arm and forearm wobbling masses to the bones used were $0.02 \mathrm{~N} / \mathrm{mm}^{3}$ and $0.03 \mathrm{~N} / \mathrm{mm}^{3}$ (Glynn et al., 2007) with corresponding near-critical damping coefficients of $0.085 \mathrm{~N} \cdot \mathrm{s} / \mathrm{mm}$ and $0.11 \mathrm{~N} \cdot \mathrm{s} / \mathrm{mm}$, respectively.

The mean RMS difference between simulation and performance for all angles in the center impact trial over the last $50 \mathrm{~ms}$ before ball impact was $0.4^{\circ}$ (Table 3 ) with an RMS difference of $0.7^{\circ}$ at the instant of ball impact (Table 3, Figure 5).

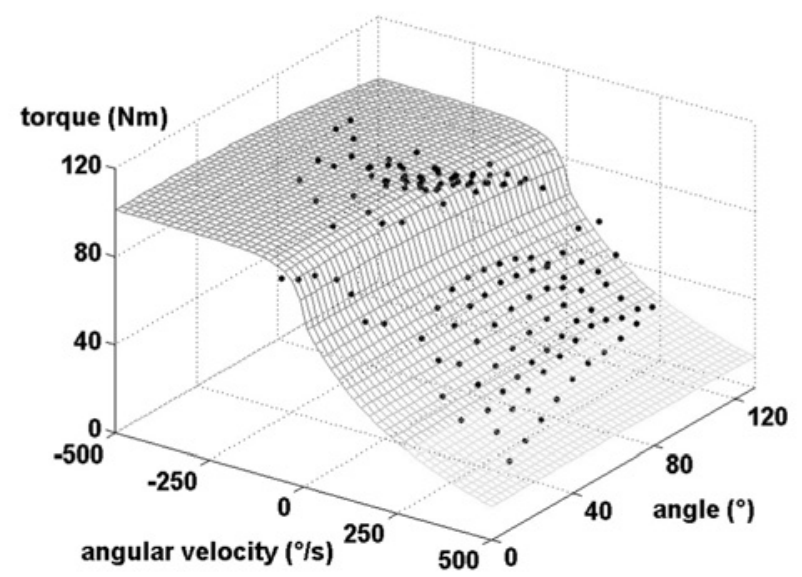

Figure 4 - Subject-specific raw torque, angle, and angular velocity data, plus the corresponding surface fit for elbow flexion.
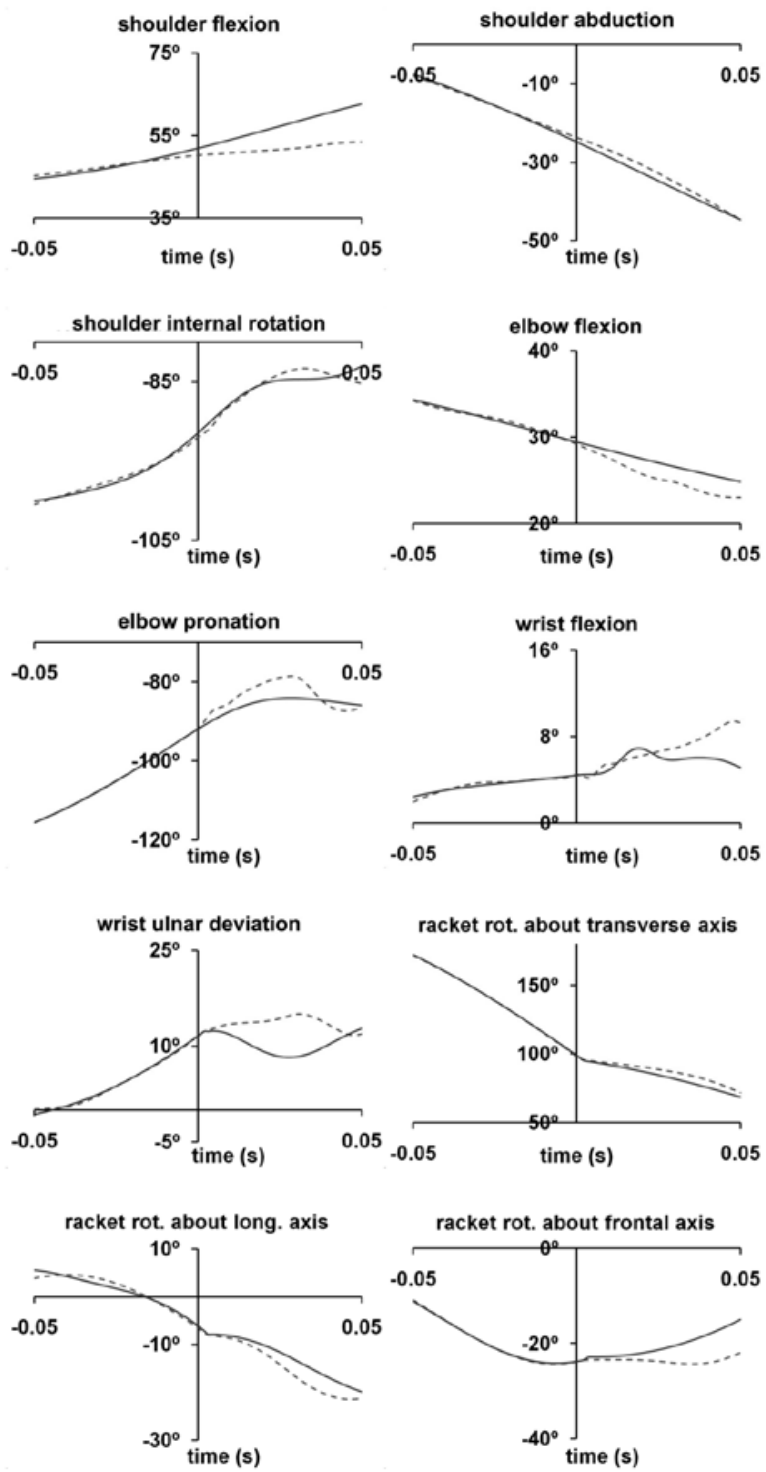

Figure 5 - A comparison of the performance (solid line) and matching simulation (dashed line) for the center impact trial. Ball impact is at time zero.

Table 1 Subject-specific segmental inertia parameters

\begin{tabular}{|c|c|c|c|c|c|c|c|c|}
\hline \multirow[b]{2}{*}{ Segment } & \multirow{2}{*}{$\begin{array}{c}\text { Mass } \\
(\mathrm{kg})\end{array}$} & \multirow{2}{*}{$\begin{array}{c}\text { Center of Mass (CM) } \\
\text { From Proximal Joint } \\
(\mathrm{mm})\end{array}$} & \multicolumn{3}{|c|}{$\begin{array}{l}\text { CM With Respect to } \\
\text { Segment's CM (mm) }\end{array}$} & \multirow{2}{*}{$\begin{array}{c}\mathrm{I}_{\mathrm{x}} \\
\left(\mathrm{kg} \cdot \mathrm{m}^{2} \cdot 10^{-3}\right)\end{array}$} & \multirow{2}{*}{$\begin{array}{c}I_{y} \\
\left(\mathrm{~kg} \cdot \mathrm{m}^{2} \cdot 10^{-3}\right)\end{array}$} & \multirow{2}{*}{$\begin{array}{c}\mathrm{I}_{\mathrm{z}} \\
\left(\mathrm{kg} \cdot \mathrm{m}^{2} \cdot 10^{-3}\right.\end{array}$} \\
\hline & & & $d_{x}(m m)$ & $d_{y}(m m)$ & $d_{z}(m m)$ & & & \\
\hline \multicolumn{9}{|c|}{ Upper Arm } \\
\hline Whole & 2.16 & 126.1 & 0.0 & 0.0 & 0.0 & 16.9 & 16.9 & 2.58 \\
\hline Humerus & 0.29 & - & -2.5 & 4.5 & 3.5 & 4.34 & 4.33 & 0.043 \\
\hline Wobbling & 1.87 & - & 0.4 & -0.7 & -0.6 & 12.6 & 12.6 & 2.52 \\
\hline \multicolumn{9}{|l|}{ Forearm } \\
\hline Whole & 1.52 & 124.4 & 0.0 & 0.0 & 0.0 & 10.2 & 10.1 & 1.21 \\
\hline Radius & 0.11 & - & 16.8 & -4.4 & -46.4 & 0.779 & 0.776 & 0.0083 \\
\hline Ulna & 0.11 & - & -4.9 & -16.5 & 17.7 & 0.751 & 0.75 & 0.0083 \\
\hline Wobbling & 1.30 & - & -1.0 & 1.8 & 2.4 & 8.33 & 8.25 & 1.12 \\
\hline Hand & 0.40 & 70.2 & 0.0 & 0.0 & 0.0 & 0.968 & 0.792 & 0.232 \\
\hline
\end{tabular}




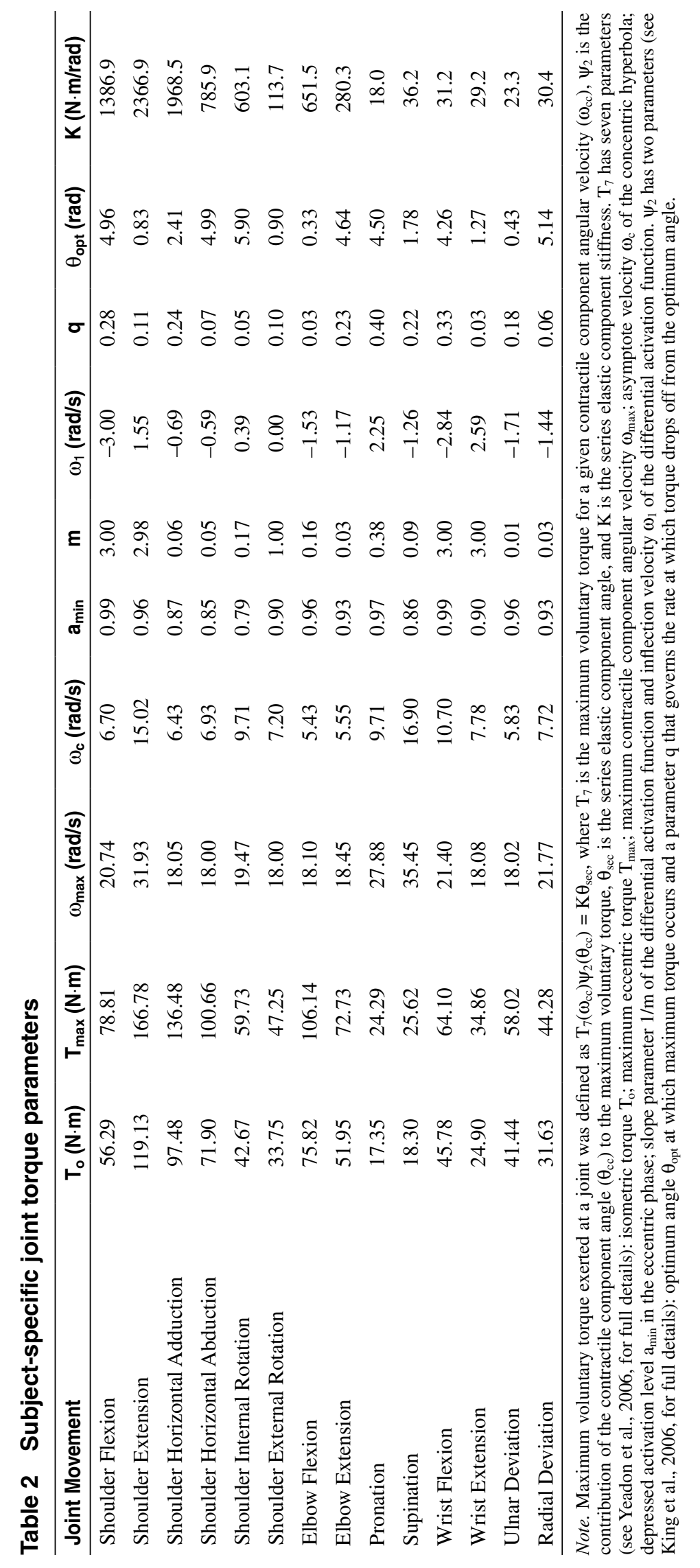


Table 3 Difference between simulation and performance for the 50-ms preimpact phase

\begin{tabular}{lcc}
\hline Joint Movement & $\begin{array}{c}\text { RMS } \\
\text { Difference }\end{array}$ & $\begin{array}{c}\text { Deviation } \\
\text { at Impact }\end{array}$ \\
\hline Shoulder Flexion/Extension & $0.73^{\circ}$ & $-1.74^{\circ}$ \\
Shoulder Abduction/Adduction & $0.46^{\circ}$ & $1.06^{\circ}$ \\
Shoulder Internal/External Rotation & $0.32^{\circ}$ & $-0.50^{\circ}$ \\
Elbow Flexion/Extension & $0.20^{\circ}$ & $-0.21^{\circ}$ \\
Pronation/Supination & $0.06^{\circ}$ & $0.18^{\circ}$ \\
Wrist Flexion/Extension & $0.15^{\circ}$ & $0.04^{\circ}$ \\
Radial/Ulnar Deviation & $0.23^{\circ}$ & $0.02^{\circ}$ \\
Racket Rotation About Transverse Axis & $0.22^{\circ}$ & $-0.66^{\circ}$ \\
Racket Rotation About Longitudinal Axis & $0.76^{\circ}$ & $-0.37^{\circ}$ \\
Racket Rotation About Frontal Axis & $0.11^{\circ}$ & $-0.02^{\circ}$ \\
\hline \multicolumn{1}{c}{ Overall RMS Difference Score } & $0.40^{\circ}$ & $0.71^{\circ}$ \\
\hline
\end{tabular}

An overall RMS difference of $2.8^{\circ}$ was obtained for the initial impact-onward matching of the center impact trial with all angle time histories matched satisfactorily. However, perturbing the ball impact location to the offcenter impact location resulted in unrealistic levels (up to $15^{\circ}$ difference) of wrist flexion and racket rotation about its longitudinal axis between the values recorded for the off-center impact performance and the off-center simulation (Figure 6, Table 4).

Modifying the matching for the center impact trial with adjusted bounds for the grip parameters resulted in a good match with a RMS difference of $3.3^{\circ}$ (Figure 5, Figure 7, Table 5, Table 6 and Table 7). In addition perturbing the ball impact location in the matching simulation to be the same as the off-center trial gave realistic results (Figure 6, Table 4). In particular, maximum wrist flexion and the rotation of the racket around its longitudinal axis improved to be within $4^{\circ}$ of the values observed from the off-center impact performance (Table 4).

\section{Discussion}

The development and evaluation of a subject-specific, torque-driven 3-D simulation model has been described with the evaluation process demonstrating that the model has sufficient complexity, without being overly complex, to accurately simulate one-handed backhand

Table 4 Maximum wrist flexion and rotation of the racket about its longitudinal axis for the off-center impact performance, original matching, and modified matching

\begin{tabular}{lcc}
\hline Variable & Wrist Flexion & Racket Rotation \\
\hline Performance & $13^{\circ}$ & $23^{\circ}$ \\
Original Matching & $22^{\circ}$ & $8^{\circ}$ \\
Modified Matching & $17^{\circ}$ & $23^{\circ}$ \\
\hline
\end{tabular}

a
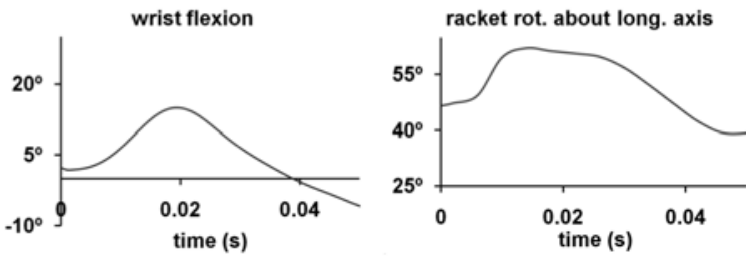

b

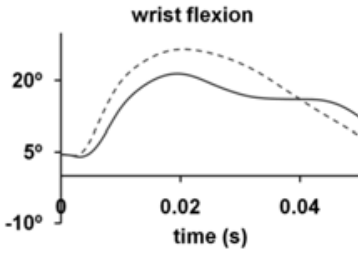

Figure 6 - Wrist flexion and longitudinal rotation of the racket for (a) the off-center performance and (b) simulation results from the center impact model perturbed to simulate offcenter impacts with initial matching (dashed line) and modified matching (solid line) parameters. Ball impact is at time zero.
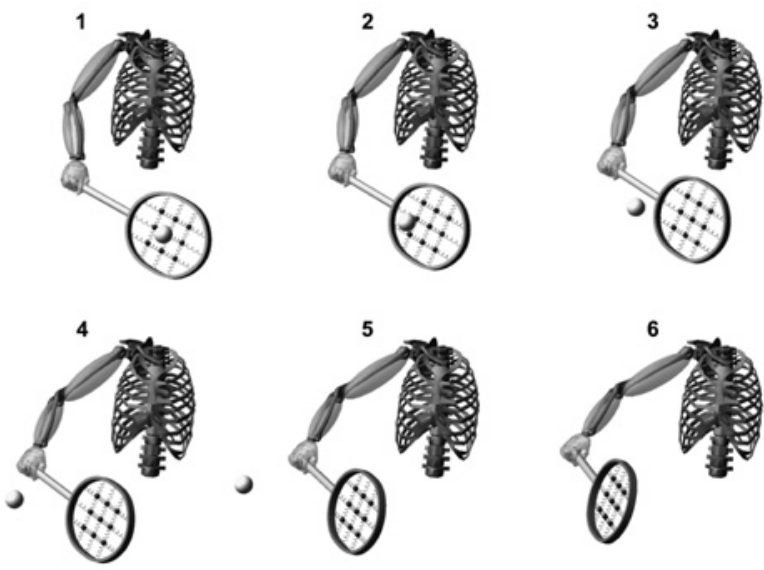

Figure 7 - Computer graphics of the matching simulation over the period from racket-ball impact until the end of the simulation (10-ms time difference between images). 
Table 5 Outbound ball velocity and contact period for the performance and the modified matching simulation

\begin{tabular}{lcc}
\hline Variable & Performance & Simulation \\
\hline $\mathrm{V}_{\text {horizontal }}(\mathrm{m} / \mathrm{s})$ & 30.9 & 27.1 \\
$\mathrm{v}_{\text {vertical }}(\mathrm{m} / \mathrm{s})$ & 6.2 & 6.2 \\
Contact Period $(\mathrm{ms})$ & 4.0 & 4.1 \\
\hline
\end{tabular}

Table 6 Difference between simulation and performance for the impact-onward phase (50 ms)

\begin{tabular}{|c|c|}
\hline Joint Movement & $\begin{array}{c}\text { RMS } \\
\text { Difference }\end{array}$ \\
\hline Shoulder Flexion/Extension & $5.96^{\circ}$ \\
\hline Shoulder Abduction/Adduction & $1.50^{\circ}$ \\
\hline Shoulder Internal/External Rotation & $0.87^{\circ}$ \\
\hline Elbow Flexion/Extension & $1.66^{\circ}$ \\
\hline Pronation/Supination & $3.30^{\circ}$ \\
\hline Wrist Flexion/Extension & $1.74^{\circ}$ \\
\hline Radial/Ulnar Deviation & $3.94^{\circ}$ \\
\hline Racket Rotation About Transverse Axis & $3.86^{\circ}$ \\
\hline Racket Rotation About Longitudinal Axis & $2.41^{\circ}$ \\
\hline Racket Rotation About Frontal Axis & $3.89^{\circ}$ \\
\hline Overall RMS Difference Score & $3.26^{\circ}$ \\
\hline
\end{tabular}

Table 7 The viscoelastic grip parameters used for the initial and modified matching simulations

\begin{tabular}{lcc}
\hline Grip Parameter & Initial & Modified \\
\hline $\mathrm{k}_{1}$ & 82.52 & 15.87 \\
$\mathrm{k}_{2}$ & 348.18 & 13.19 \\
$\mathrm{k}_{3}$ & 44.05 & 4.68 \\
$\mathrm{c}_{1}$ & 3.25 & 0.86 \\
$\mathrm{c}_{2}$ & 1.50 & 13.57 \\
$\mathrm{c}_{3}$ & 4.53 & 0.25 \\
\hline
\end{tabular}

Note. Grip torque component: $T=-k \theta-c \theta \dot{\theta}_{i}$, where $\mathrm{i}=1,2$, 3 corresponds to the longitudinal, frontal, and transverse axes of the tennis racket respectively, $\theta_{\mathrm{i}}=$ the racket angle relative to the position at impact, $\mathrm{k}_{\mathrm{i}}$ and $\mathrm{c}_{\mathrm{i}}=$ the stiffness $(\mathrm{N} \cdot \mathrm{m} / \mathrm{rad})$ and damping $\left(\mathrm{N} \cdot \mathrm{m} \cdot \mathrm{s} / \mathrm{rad}^{2}\right)$ coefficients respectively.

ground strokes from an elite tennis player. The model was evaluated by matching a recorded center impact one-handed backhand stroke with the resulting matching simulation giving a good match to both a center impact trial and an off-center impact trial (Tables 5 and 6, and Figure 7). Requiring the center impact matching simulation to behave realistically when the ball impact location was perturbed to an off-center location was challenging but necessary, as this equates to the real-life situation where the player uses a preprogrammed technique and does not have time to react to an off-center impact and change technique until later than the first $50 \mathrm{~ms}$ after ball-racket impact.

The simulation model described has sufficient complexity, without being overly complex, to address questions regarding the factors that contribute to increased levels of eccentric contraction of the wrist extensors during one-handed tennis backhand ground strokes. In particular, simulations can be run to isolate and investigate the effect of ball impacts at a range of locations on the stringbed, the effect of varying levels of grip torque, the effect of different racket characteristics and stringbed tensions, and the effect of different player techniques. Areas in which the complexity of the model could have been increased but was not include the torque activation profiles: simple ramp on / ramp off profiles were used because these proved sufficient to match the performance trials satisfactorily. Increasing the complexity of the activation profiles may have improved the matching of the performance data toward the end of the 50-ms simulation period, but because the most critical period for this study was around $25 \mathrm{~ms}$ after impact, this increase in complexity was deemed unnecessary. Furthermore, a realistic but not overly complex 3 rotational degree-offreedom grip representation has been used in this study, whereas Nesbit et al. (2006) assumed a rigid connection between the racket and hand and Glynn et al., (2007) used a 6 degree-of-freedom representation. The model evaluation carried out in this study suggested that a 3 rotational degree-of-freedom joint was sufficient and showed that a rigid connection between hand and racket was unrealistic. This is consistent with the experimental studies on the coupling of the hand and racket in which it has been demonstrated that a rigid connection is not realistic (Brody, 1989; Hatze, 1992; Kawazoe, 1997; Knudson, 1997; Hennig, 2007).

Evaluating a simulation model before it is used is a crucial and vital part of the process, which is required so that confidence can be gained in the results obtained from simulations. In addition, this study has demonstrated that, wherever possible, consideration of the models response to perturbations should be accounted for within the evaluation process so that confidence can be obtained in the results obtained. In particular, although good agreement was found between the center impact performance and the initial matching simulation, when the location of ball impact was perturbed to an off-center location, the resulting simulation demonstrated much larger wrist flexion and relatively little rotation of the racket within the hand after impact when compared with the equivalent off-center trial. This initially unrealistic off-center simulation was due to the torsional grip tightness parameters being too high and not allowing sufficient relative rotation of the racket within the hand. Perturbing grip tightness demonstrated that the center-impact simulation was not particularly sensitive to the grip parameters, as there is little tendency for the racket to rotate around its 
longitudinal axis when the ball contacts the center of the stringbed. As a consequence, the initial matching optimization failed to give realistically low grip tightness parameters, as there was little information in the performance data of the center impact to help quantify them (Table 7). Generally, using a matching process to determine some model parameters will give good estimates for those parameters that have a marked effect on the RMS difference score but give less good estimates for those parameters that the activity modeled is less sensitive to. The evaluation process has demonstrated the relationship between grip tightness / ball impact location and the motion of the racket/arm after impact for an elite subject with the results consistent with experimental studies (Hatze, 1976; Hennig et al., 1992). In particular, forced wrist flexion linked to off-center impacts observed under experimental conditions (Knudson \& Blackwell, 1997; Riek et al., 1999) was found to be related to grip tightness, with a tighter grip resulting in more forced wrist flexion and less rotation of the racket within the hand compared with a looser grip.

Although the model has been successfully evaluated for a single subject and is in line with the evaluations of previously developed simulation models (Yeadon \& King, 2002; King et al., 2006), it would be advantageous to evaluate the model for additional subjects in the future. For example, investigating the effects of major changes such as the type of stroke (topspin vs. backspin) or experience level (elite vs. novice), it would be prudent to reevaluate the model and obtain new matching simulations as the dynamics of the stroke and conditions at impact would be considerably different. In addition, the model could be used to help understand the large variability in postimpact kinematics and kinetics that have been observed in experimental studies, along with further work incorporating variability in strength, and the skill level of different subjects within the model.

The main advantage of the model developed in this study over previous simulations of tennis strokes (e.g., Nesbit et al. (2006) and Glynn et al. (2007)) is that the simulation model is torque driven. As a consequence, the model can be used to address a wider range of questions and simulate a range of shots and conditions, rather than be limited essentially to movements that have been recorded. For example, the model can be used to investigate the effect of racket parameters, technique and ball impact location on the motion of the racket arm / racket during a tennis stroke. These sorts of investigations cannot be fully addressed using angle-driven models, as the technique is fixed to that of actual performances, and, in addition, they are difficult to carry out experimentally since it is not possible to vary a single factor without other factors changing as well (Yeadon \& Challis, 1994). In the future, the simulation model presented in this study could be adapted so that it is muscle driven. This would be a nontrivial step that requires many more parameters to be determined and as a consequence it would be much harder to produce a subject-specific model that can be evaluated by comparison with actual performances.
Furthermore, the interface between the racket and hand could be examined in more detail and this might allow a more in-depth analysis into the effect of grip torque on key injury-related variables.

A torque-driven forward dynamics simulation model has been developed and shown to give realistic simulations for center and off-center one-handed tennis backhand strokes. The model can be used to simulate ball impacts at a range of locations on the stringbed as well as to investigate the effect of perturbations of other model variables on the levels of eccentric contraction of the wrist extensors during one-handed tennis backhand ground strokes. Grip tightness and ball impact location are clearly important parameters that affect tennis racket / arm kinematics and these should be investigated further to understand the interaction between racket and arm during tennis strokes with particular reference to the levels of eccentric contraction of the wrist extensors.

\section{Acknowledgments}

The authors would like to acknowledge the financial support provided by the Head Austria GmbH and U.K. EPSRC.

\section{References}

Bauer, J.A., \& Murray, R.D. (1999). Electromyographic patterns of individuals suffering from lateral tennis elbow. Journal of Electromyography and Kinesiology, 9(4), 245-252.

Blackwell, J.R., \& Cole, K.J. (1994). Wrist kinematics differ in expert and novice tennis players performing the backhand stroke: Implications for tennis elbow. Journal of Biomechanics, 27(5), 509-516.

Brody, H. (1989). Vibration Damping of Tennis Rackets. International Journal of Sport Biomechanics, 5(4), 451-456.

Carroll, R. (1981). Tennis elbow: incidence in local league players. British Journal of Sports Medicine, 15(4), 250-256.

Chow, J.W., Carlton, L.G., Lim, Y.T., Shim, J.H., Chae, W.S., \& Kuenster, A.F. (1999). Muscle activation during the tennis volley. Medicine and Science in Sports and Exercise, 31(6), 846-854.

Clarys, J.P., \& Marfell-Jones, M.J. (1986). Anthropometric prediction of component tissue masses in the minor limb segments of the human body. Human Biology, 58(5), 761-769.

Corana, A., Marchesi, M., Martini, C., \& Ridella, S. (1987). Minimizing multimodal functions of continuous-variables with the simulated annealing algorithm. ACM Transactions on Mathematical Software, 13(3), 262-280.

de Rugy, A., \& Sternad, D. (2003). Interaction between discrete and rhythmic movements: reaction time and phase of discrete movement initiation during oscillatory movements. Brain Research, 994(2), 160-174.

de Zee, M., \& Voigt, M. (2001). Moment dependency of the series elastic stiffness in the human plantar flexors measured in vivo. Journal of Biomechanics, 34(11), 1399-1406.

Giangarra, C.E., Conroy, B., Jobe, F.W., Pink, M., \& Perry, J. (1993). Electromyographic and cinematographic analysis of elbow function in tennis players using single- and double-handed backhand strokes. American Journal of Sports Medicine, 21(3), 394-399. 
Glynn, J.A., King, M., \& Mitchell, S.R. (2007). A computer simulation of one-handed backhand strokes to investigate elbow joint loading. In S. Miller and J. Capel-Davies (Eds.), Tennis Science and Technology 3, 129-133. London: ITF

Hatze, H. (1976). Forces and duration of impact, and grip tightness during the tennis stroke. Medicine and Science in Sports and Exercise, 8(2), 88-95.

Hatze, H. (1992). Objective Biomechanical Determination of Tennis Racket Properties. International Journal of Sport Biomechanics, 8(4), 275-287.

Hennig, E.M., Rosenbaum, D., \& Milani, T.L. (1992). Transfer of tennis racket vibrations onto the human forearm. Medicine and Science in Sports and Exercise, 24(10), 1134-1140.

Hennig, E.M. (2007). Influence of Racket Properties on Injuries and Performance in Tennis. Exercise and Sport Sciences Reviews, 35(2), 62-66.

Kawazoe, Y. (1997). Experimental Identification of a Hand-Held Tennis Racket and Prediction of Rebound Ball Velocity in an Impact. In G. Yagawa \& C. Miki (Eds.), Theoretical and Applied Mechanics (Vol. 46, pp. 177-188). Tokyo: Nctam.

Kelley, J.D., Lombardo, S.J., Pink, M., Perry, J., \& Giangarra, C.E. (1994). Electromyographic and cinematographic analysis of elbow function in tennis players with lateral epicondylitis. American Journal of Sports Medicine, 22(3), 359-363.

King, M.A., \& Yeadon, M.R. (2002). Determining subjectspecific torque parameters for use in a torque-driven simulation model of dynamic jumping. Journal of Applied Biomechanics, 18(3), 207-217.

King, M.A., Wilson, C., \& Yeadon, M.R. (2006). Evaluation of a torque-driven model of jumping for height. Journal of Applied Biomechanics, 22(4), 264-274.

Knudson, D. (1997). Effect of grip models on rebound accuracy of off-center tennis impacts. In J. Wilkerson, K. Ludwig, and W. Zimmermann (Eds.), Biomechanics in Sports XV: Proceedings of the 15th International Symposium on Biomechanics in Sports, 483-487. Denton, TX.

Knudson, D., \& Blackwell, J. (1997). Upper Extremity Angular Kinematics of the One-Handed Backhand Drive in Tennis Players With and Without Tennis Elbow. International Journal of Sports Medicine, 18(2), 79-82.

Knudson, D. (2004). Biomechanical studies on the mechanism of tennis elbow. In M. Hubbard, R.D. Mehta, \& J.M. Pallis (Eds.), The Engineering of Sport 5 (Vol. 1, pp. 135-141). Sheffield: International Sports Engineering Association.

Knudson, D.V. (1991). Forces on the Hand in the Tennis One-Handed Backhand. International Journal of Sport Biomechanics, 7(3), 282-292.

Lewis, R.D., \& Brown, J.M.M. (1994). Influence of muscle activation dynamics on reaction-time in the elderly. European Journal of Applied Physiology and Occupational Physiology, 69(4), 344-349.
McLaughlin, T.M., \& Miller, N.R. (1980). Techniques for the evaluation of loads on the forearm prior to impact in tennis strokes. Journal of Mechanical Design, 102, 701-710.

Morrey, B.F., \& Chao, E.Y.S. (1976). Passive motion of elbow joint - Biomechanical analysis. Journal of Bone and Joint Surgery, 58-A(4), 501-508.

Neptune, R.R., McGowan, C.P., \& Fiandt, J.M. (2009). The Influence of Muscle Physiology and Advanced Technology on Sports Performance. Annual Review of Biomedical Engineering, 11, 81-108.

Nesbit, S.M., Elzinga, M., Herchenroder, C., \& Serrano, M. (2006). The effects of racket inertia tensor on elbow loadings and racket behavior for central and eccentric impacts. Journal of Sports Science and Medicine, 5, 304-317.

Pain, M.T.G., \& Challis, J.H. (2001). The role of the heel pad and shank soft tissue during impacts: a further resolution of a paradox. Journal of Biomechanics, 34(3), 327-333.

Pluim, B.M., Staal, J.B., Marks, B.L., Miller, S., \& Miley, D. (2007). Health benefits of tennis. British Journal of Sports Medicine, 41(11), 760-768.

Riek, S., Chapman, A.E., \& Milner, T. (1999). A simulation of muscle force and internal kinematics of extensor carpi radialis brevis during backhand tennis stroke: implications for injury. Clinical Biomechanics (Bristol, Avon), 14(7), 477-483.

Roetert, E.P., Brody, H., Dillman, C.J., Groppel, J.L., \& Schultheis, J.M. (1995). The Biomechanics of Tennis Elbow - an Integrated Approach. Clinics in Sports Medicine, 14(1), $47-57$.

Wood, G.A., \& Jennings, L.S. (1979). Use of Spline Functions for Data Smoothing. Journal of Biomechanics, 12(6), 477-479.

Yeadon, M.R. (1990). The simulation of aerial movement - II: A mathematical inertia model of the human body. Journal of Biomechanics, 23, 67-74.

Yeadon, M.R., Atha, J., \& Hales, F.D. (1990). The simulation of aerial movement - IV A computer-simulation model. Journal of Biomechanics, 23(1), 85-89.

Yeadon, M.R., \& Challis, J.H. (1994). The future of performance-related sports biomechanics research. Journal of Sports Sciences, 12(1), 3-32.

Yeadon, M.R., \& Hiley, M.J. (2000). The mechanics of the backward giant circle on the high bar. Human Movement Science, 19(2), 153-173.

Yeadon, M.R., \& King, M.A. (2002). Evaluation of a torquedriven simulation model of tumbling. Journal of Applied Biomechanics, 18, 195-206.

Yeadon, M.R., King, M.A., \& Wilson, C. (2006). Modelling the maximum voluntary joint torque/angular velocity relationship in human movement. Journal of Biomechanics, 39(3), 476-482. 